\title{
Association between hepatitis $B$ virus infection status and diabetes in over 10 million Chinese women $20-49$ years
}

Ying Yang ${ }^{1}$, Fang Zhao ${ }^{2}$, Long Wang ${ }^{3}$, Tong $\mathrm{Li}^{4}$, Zuoqi Peng ${ }^{1}$, Hui Zhuang ${ }^{4}$, Puhong Zhang ${ }^{2}$, Xu Ma ${ }^{1}$.

${ }_{1}^{1}$ National Research Institute for Family Planning, China DOHaD Research Center- National Human Genetic Resources Center, Beijing, China.

2 The George Institute for Global Health at Peking University Health Science Center, Beijing, China.

${ }^{3}$ Graduate School of Peking Union Medical College, Beijing, China.

${ }^{4}$ Peking University Health Science Center, Department of Microbiology and Infectious Disease CenterSchool of Basic Medical Sciences, Beijing, China.

\section{Background}

Previous studies had identified an association between hepatitis $B$ virus (HBV) infection and the prevalence of diabetes mellitus (DM). However, the results of those studies were not consistent.

Aim We aimed to examine the association between HBV infection and DM in a nationwide population who are Chinese women aged 20-49 years old preparing for pregnancy.

\section{Method}

This cross-sectional study included 11201231 females from the National Free Pre-pregnancy Checkup Projects conducted across 31 provinces in China in 2014-2015. DM was defined as self-reported history of diabetes or current use of antidiabetic medications or fasting plasma glucose (FPG) levels $\geq 126 \mathrm{mg} / \mathrm{dL}$ (7.0 mmol/L). Impaired Fasting Glucose (IFG) was defined as FPG levels of $100 \mathrm{mg} / \mathrm{dL}(5.6 \mathrm{mmol} / \mathrm{L})$ to $125 \mathrm{mg} / \mathrm{dL}(6.9$ $\mathrm{mmol} / \mathrm{L})$. HBV infection status was categorized into six groups according to the different results of HBV markers, including HBsAg, anti-HBs, HBeAg, anti-HBe and anti-HBc, test as follow: A. negative for all the HBV markers (called susceptible group); B. anti-HBs positive alone (called immunized group); C. HBsAg positive, anti-HBs negative, $\mathrm{HBeAg}$ positive, anti-HBe negative and anti-HBc positive or negative (called high-replicative group); D. HBsAg positive, anti-HBs negative, $\mathrm{HBeAg}$ negative, anti-HBe positive and anti-HBc positive or negative (called low-replicative group); $\mathrm{E}$. HBsAg negative, $\mathrm{HBeAg}$ negative, anti-HBe negative, anti-HBc positive, and anti-HBs positive or negative (called resolved group); F. others. Both univariate and multivariate logistic regression were performed to examine the associations between HBV infection and DM or IFG and DM.

\section{Results}

Univariate analysis results showed that women with $\mathrm{HBsAg}$ positive had a higher prevalence of diabetes (1.8\% vs $1.3 \%$ ), meanwhile the prevalence of diabetes in HBV immunized women or susceptible population was almost the same (1.3\%). As for women in HBV infection low-replicative group or resolved group, the prevalence of DM was higher than the susceptible population, even after adjusting all potential confounding variables. Women in HBV infection high-replicative group showed a positive association between HBV infection and DM, however, it turned to be inversely associated with DM when the ALT level was adjusted in multivariable analyses $(\mathrm{OR}=1.293$ (1.241-1.346) vs 0.918 (0.881-0.957). (See figure 1)

\section{Discussion}

To our knowledge, it was the first report of using the five HBV markers to examine the association between HBV infection status and DM. Our findings showed that there was a strong association between HBV infection and DM, no matter the stage of infection in a nationwide women population, which indicated HBV infection may have a predisposing effect on the development of DM. In immunized group, the OR was 0.986 (0.973-0.998), which indicated a protective effect of HBV immunization to the development of DM. This finding was consistent with a recent study which was derived from a representative US population. Their result showed successful HBV vaccination was associated with a reduced OR of diabetes (0.67, 95\% Cl: 0.52-0.84). Result from 900 retired Chinese workers also found a strong negative correlation between positive HBsAb status and the presence of diabetes in female participants. Both studies implicated diabetes risk deduction by successful vaccination. As known to all, both HBV infection and diabetes were major global public health problems, especially in China. For public health, successful HBV vaccination was not only benefit to prevent HBV infection but also could have a significant impact on the epidemic of diabetes. An intervention trial is warranted before promoting a large scale application of HBV vaccination for prevention of diabetes.

Figure 1. Association between hepatitis B virus infection and IFG/DM ----results of multivariate logistic regression analyses.

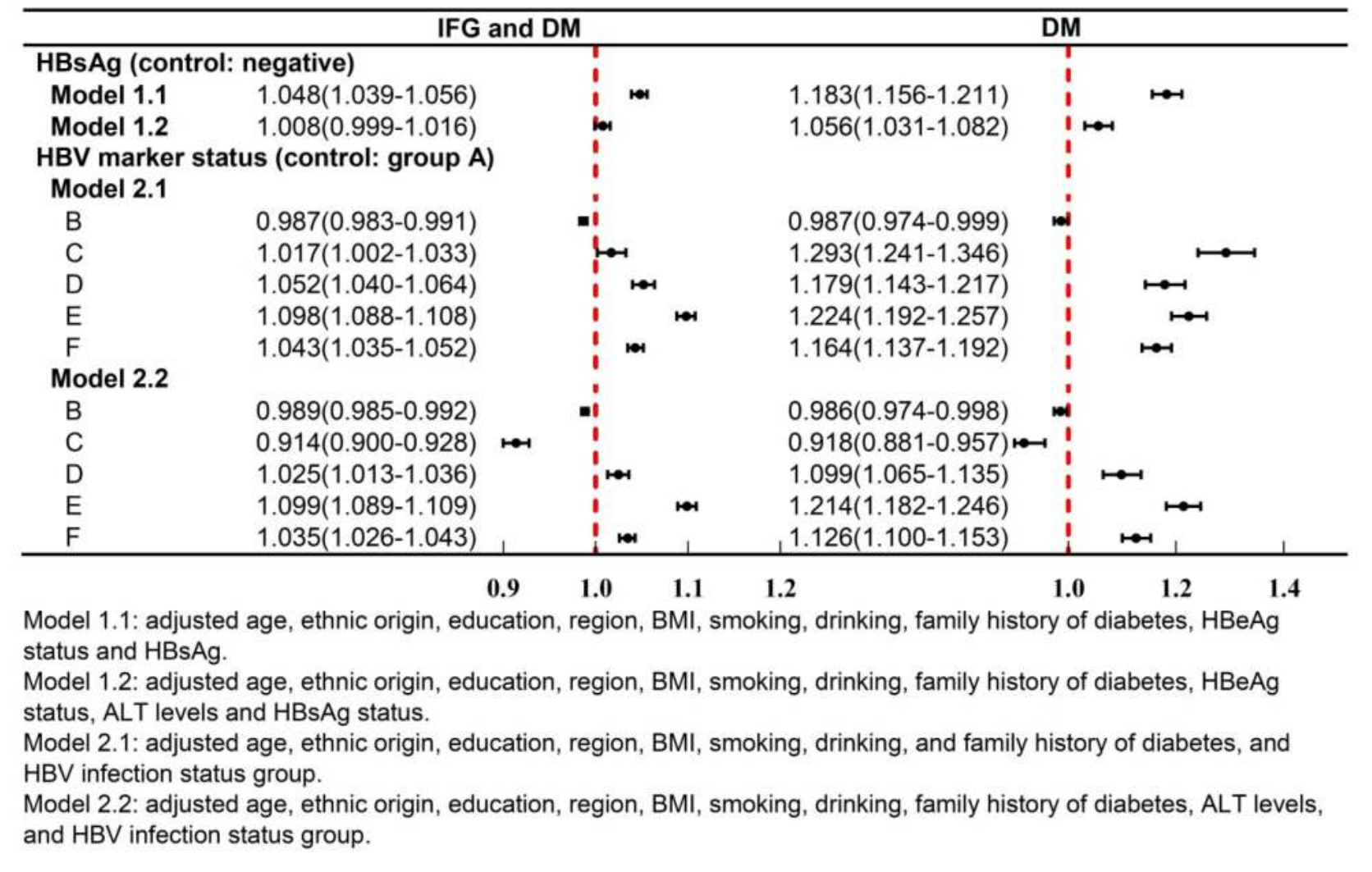

